\title{
Continuous lasing for Perovskites
}

Optically generated local phase changes produce a transient quantum well structure with robust optical gain. The work may give important directions for the development of a printable electrically pumped laser.

Solution-processable semiconductors promise to bring to optoelectronics what 3D printing may bring to mechanical engineering - a low-cost and energy-efficient way to manufacture components with a maximum of flexibility to adjust design and function. Organic materials with $\pi$-conjugated electron systems have been leading the way here. Over the past decade, information displays based on organic light-emitting diodes (OLEDs) have entered mass production, even though most commercial products are currently produced using high-vacuum rather than solution processing. Likewise, organic photovoltaics and organic transistors have made great strides. More recently, trihallide perovskites - a hybrid between organic and inorganic semiconductor - have thoroughly stirred up the photovoltaics community, with the energy conversion efficiency of perovskite solar cells now rivalling multi-crystalline silicon. One major device that is still lacking in solution processed thin film optoelectronics is the electrically pumped laser. Due to the widespread use of lasers in information, telecommunication, manufacturing and sensing technology, the development of an electrically pumped laser is expected to unlock a wide range of new applications for solutionprocessed semiconductors and over the past two decades has become the 'holy grail' of the field.

As Chris Giebink, Barry Rand and co-workers report on p. xx of this issue ${ }^{1}$, perovskites may bring us closer to finally reaching this goal. While the latest work does not demonstrate an electrically pumped perovskite laser, the scientists have succeeded in realizing true continuous wave (c.w.) operation from a laser based on the perovskite methylammonium lead iodide $\left(\mathrm{MAPbl}_{3}\right)$. Under constant optical pumping by a blue light-emitting diode, their devices emit $785 \mathrm{~nm}$ wavelength laser light non-stop for up to an hour, at least when kept at a temperature of $\approx 100 \mathrm{~K}$. In order to achieve c.w. lasing, the scientists use a clever trick to dynamically generate exciton-trapping sites in the material and thus mitigate or at least reduce some of the issues encountered in many solid-state laser materials. Earlier organic and perovskite lasers have generally required excitation with nanosecond or shorter light pulses to avoid thermal runaway and manage build-up of parasitic species in the material - such as triplet excitons in organic semiconductors. These species otherwise tend to interfere with the lasing process by introducing absorption at the lasing wavelength or by bleaching the lasing transition.

C.w. operation is often regarded as a stepping-stone to the development of electrically driven lasers, in particular in the organic laser community. While the technical challenges involved in both differ, there are important links: Nanosecond or sub-nanosecond electrical pumping is technically challenging to achieve, in particular because the low charge carrier mobility and short excited-state lifetime of many organic materials will likely require current densities in the $\mathrm{kA} / \mathrm{cm}^{2}$ range or higher to achieve population inversion and thus realize lasing. In addition, sustaining such high excitation rates with technically feasible electrical pumping schemes or by continuous optical pumping will likely require careful heat management to avoid thermal runaway. Furthermore, the high exciton densities required for population inversion tend to incur non-linear annihilation effects. For many materials, including perovskites and colloidal quantum dots - a further contender to the solutionprocessed thin-film electrically driven laser ${ }^{2}-$, Auger recombination is of particular relevance. In conventional inorganic semiconductors Auger recombination is generally controlled by epitaxial growth of quantum well or quantum dot hetero-structures (Fig. 1a). In organic materials, exciton- 
exciton annihilation needs to be carefully managed, e.g. by mixing a small amount of the molecules that provide optical gain (guest molecules) into a larger exciton energy host matrix to separate the guest molecules from each other (typically guest concentrations are $\approx 1 \%$, Fig. $1 \mathrm{~b}$ ) ${ }^{3}$. In fluorescent proteins - a naturally occurring emitter molecule and gain material -, annihilation is mitigated via an intricate molecular bumper structure that adjusts the distance between emissive sites (Fig. 1c) ${ }^{4}$.

A further important issue are triplet states. Three quarters of the excited states formed via electrical injection have triplet character. In organic materials, the radiative decay of triplets is generally spinforbidden and hence extremely slow (microseconds or longer), which means that once a molecule is in the triplet state it is effectively no longer available for stimulated emission. Furthermore, absorption of triplet states often introduces considerable optical loss at the intended lasing wavelength. During continuous optical pumping, a fraction of the singlet excitons transitions to the energetically lower triplet manifold by intersystem crossing and the resulting triplet accumulation has so far been the principal obstacle to realizing a solid-state c.w. laser based on pure organic semiconductors. In bulk perovskites, strong spin-orbit coupling and a small exciton binding energy (typically, tens of meV) frustrate spin-conservation, similar to the situation in conventional inorganic semiconductors. This means that triplet accumulation is a lesser issue for perovskite-based lasers. Very recent work even argues that at least for certain perovskites the lowest energy excited triplet state is a "bright" state - that is a state with an allowed transition to the ground state - whereas the energetically higher singlet state is predicted to be "dark" ${ }^{5}$. For two-dimensional perovskites, which offer a range of interesting electro-optical properties, excitons are more strongly bound and it has been found that for these the triplet states only have weakly allowed radiative transitions (lifetime, $10-100 \mathrm{~ns})^{6,7}$.

When the $\mathrm{MAPbl}_{3}$ that is used as laser gain material in the new work is cooled down to below about $160 \mathrm{~K}$, it undergoes a phase transition from a tetragonal to an orthorhombic lattice structure, with the latter having a slightly larger optical bandgap. The researchers fabricated a distributed feedback laser structure based on $\mathrm{MAPbl}_{3}$ and cooled it down to $\approx 100 \mathrm{~K}$, i.e. well into the orthorhombic phase. Upon excitation with intense blue light $\left(>17 \mathrm{~kW} / \mathrm{cm}^{2}\right)$, tetragonal phase inclusions formed inside the orthorhombic lattice within a few $100 \mathrm{~ns}$, presumably due to local heating (Fig. 1d). This led to a transient energy landscape for excitons that is similar to a quantum well structure or an organic host-guest mixture. Although $<1 \%$ of the material changed into the tetragonal phase, the emission red-shifted from $750 \mathrm{~nm}$ (characteristic of the orthorhombic phase) to $785 \mathrm{~nm}$ (characteristic of the tetragonal phase). The existence of transient low bandgap tetragonal phase inclusions in orthorhombic $\mathrm{MAPbl}_{3}$ was already reported in earlier reports and it has been hypothesized that they may be exploited to improve laser performance ${ }^{8,9}$. The present work now finds that while optical gain and lasing in bulk tetragonal $\mathrm{MAPbl}_{3}$ cease within less than $100 \mathrm{~ns}$, the mixed phase structure can sustain continuous lasing. In the current implementation, lasing lasts for up to an hour; limited by a slow blue shift of the gain spectrum away from the resonance wavelength of the distributed feedback structure.

The mechanism that enables c.w. lasing in this special phase mixture is unfortunately not yet clear. A possible explanation is that tetragonal phase inclusions act as charge carrier sink, thus isolating excitons on different inclusions from each other and locally increasing population inversion. In addition, the orthorhombic phase is likely to exert mechanical strain on the tetragonal phase inclusions and there is evidence that this strain can increase the radiative rate and emission crosssection of tetragonal phase $\mathrm{MAPbl}_{3}{ }^{10}$.

While the new report provides clear evidence for c.w. lasing, a number of important challenges remain. Most importantly, the need to work close to the orthorhombic-to-tetragonal phase 
transition temperature limits the potential for practical applications and in addition leads to variable laser characteristics. The authors find that the lasing threshold and emission wavelength depend on the thermal history of the laser and the pump power, respectively, and thus change over time. As the phase inclusions form due to pump induced heating, the maximum pump intensity and hence output power of a laser based on this design is likely limited. Finally, it is not clear whether the phase inclusion trick can also work effectively in other perovskites, e.g. in materials that emit in a different spectral range. None of the above points diminishes the value of the present proof-of-concept demonstration. The fact that phase inclusions are generated dynamically could even be used to form transient and spatially varying gain profiles across the sample. This may lead to exciting possibilities, e.g. the ability to reconfigure beam-shape and lasing wavelength, or may even allow creating optical switches and novel tools to study the physics of coupled lasers. However, along the way toward a practically useful and potentially electrically pumped perovskite laser, the phase transition concept will likely need to be optimized further. In principle, to relax the cooling requirement, one can attempt to increase the orthorhombic-to-tetragonal phase transition temperature or look for other phase transitions that also facilitate spontaneous formation of small band gap inclusions surrounded by a phase with larger band-gap. For general applications of perovskite lasers, it will likely be more convenient to copy only the design principle of the quantum well type structure. Instead of relying on a dynamic phase change process, it may be possible to achieve a similar effect by designing suitable material blends or by reducing the dimensionality of the material. Whichever strategy proves successful in the end, careful control of local defect states has once more proven to be a powerful tool to advance optoelectronics.

Malte C. Gather is at SUPA School of Physics and Astronomy, University of St Andrews, North Haugh, St Andrews KY16 9SS, UK.

e-mail:mcg6@st-andrews.ac.uk

\section{References}

${ }^{1}$ Y. Jia, R.A. Kerner, A.J. Grede, B.P. Rand, and N.C. Giebink, Nat. Photonics XX, XX (2017).

${ }^{2}$ F. Fan, O. Voznyy, R.P. Sabatini, K.T. Bicanic, M.M. Adachi, J.R. McBride, K.R. Reid, Y.-S. Park, X. Li, A. Jain, R. Quintero-Bermudez, M. Saravanapavanantham, M. Liu, M. Korkusinski, P. Hawrylak, V.I. Klimov, S.J. Rosenthal, S. Hoogland, and E.H. Sargent, Nature 544, 75 (2017).

${ }^{3}$ A.J.C. Kuehne and M.C. Gather, Chem. Rev. 116, 12823 (2016).

${ }^{4}$ C.P. Dietrich, A. Steude, L. Tropf, M. Schubert, N.M. Kronenberg, K. Ostermann, S. Höfling, and M.C. Gather, Sci. Adv. 2, e1600666 (2016).

${ }^{5}$ M.A. Becker, R. Vaxenburg, G. Nedelcu, P.C. Sercel, A. Shabaev, M.J. Mehl, J.G. Michopoulos, S.G. Lambrakos, N. Bernstein, J.L. Lyons, T. Stöferle, R.F. Mahrt, M. V. Kovalenko, D.J. Norris, G. Rainò, and A.L. Efros, arXiv 1707.03071 (2017).

${ }^{6}$ N. Kitazawa and Y. Watanabe, J. Phys. Chem. Solids 71, 797 (2010).

${ }^{7}$ T. Goto, H. Makino, T. Yao, C.H. Chia, T. Makino, Y. Segawa, G.A. Mousdis, and G.C. Papavassiliou, Phys. Rev. B 73, 115206 (2006).

${ }^{8}$ C. Wehrenfennig, M. Liu, H.J. Snaith, M.B. Johnston, and L.M. Herz, APL Mater. 2, 81513 (2014).

${ }^{9}$ S. Neutzner, A. Ram, S. Kandada, G. Lanzani, and A. Petrozza, J. Phys. Chem. C 4, 4630 (2016).

${ }^{10}$ T. Wang, B. Daiber, J.M. Frost, S.A. Mann, E.C. Garnett, A. Walsh, and B. Ehrler, Energy Environ. Sci. 
10, 509 (2017).

a

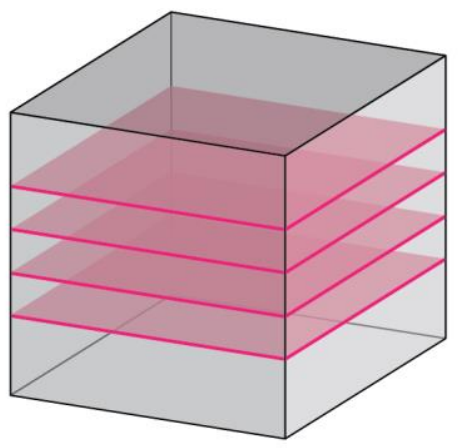

C

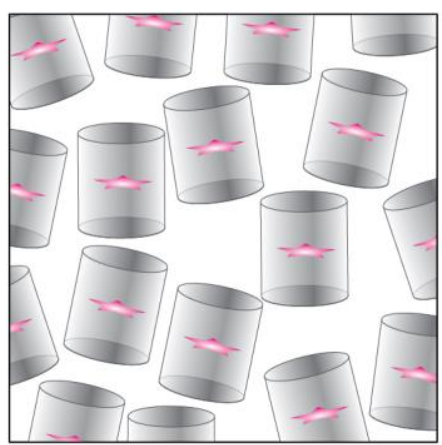

b

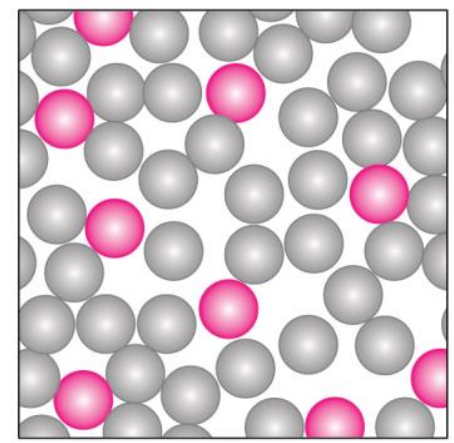

d

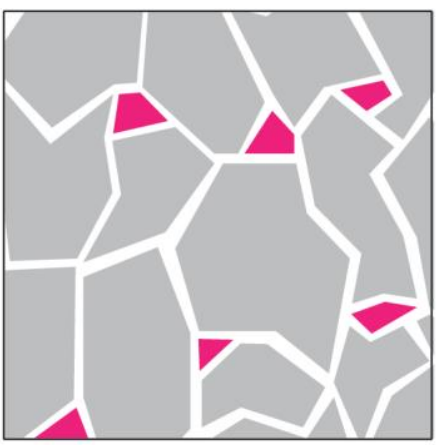

Fig. 1. Spatially separated local defect states facilitate high laser performance in different material platforms. a, Conventional inorganic semiconductor quantum well heterostructure (emissive regions in pink, passive regions in grey). b, In organic lasers, host molecules prevent exciton-exciton annihilation on the gain-providing guest molecules and in some cases act as optical antennae for pump light. c, Naturally occurring fluorescent protein molecules have a cylindrical barrel structure that ensures optimal separation between the emissive moieties located at the centre of each molecule. $\mathbf{d}$, In the new work, transient photo-generated phase inclusions in a predominantly orthorhombic perovskite provide stable optical gain. 